\title{
Care of HIV-infected patients in China
}

\author{
Yun Zhen CAO ${ }^{1, *}$, Hong Zhou LU ${ }^{2}$ \\ ${ }^{1}$ The AIDS Research Center, Chinese Academy of Medical Sciences Peking Union Medical College, Beijing 100730, China \\ ${ }^{2}$ Department of Infectious Diseases, Shanghai/FuDan Public Health Center \& Huashan Hospital, Fudan University, Shang- \\ hai 201508, China
}

\begin{abstract}
Compared with high infection areas of the world, the total HIV infection rate in China is relatively low. Nonetheless, because of China's vast territory and large population, the potential infection risk must be taken seriously. In the next few years, needle sharing among injection drug users will remain the most common route of transmission for the HIV/ AIDS epidemic in China. Unprotected sex is gradually becoming a major route of transmission. China began to implement HAART in 1999 according to international standards. Prior to 2003, there were only about 150 HIV/AIDS patients were treated with HAART in some clinical trials and about 100 HIV/AIDS patients were treated by private sources. Results of those treatments are the scientific basis for development of the therapeutic strategies in China. In March of 2003, the Chinese government initiated China CARES program. In November of 2003, the Chinese Ministry of Health announced a national policy of free ARV treatment to all HIV+ Chinese citizens who were in poverty and required ARV therapy. There are total of 19,456 HIV/AIDS patients received free ARV drugs to date in 159 regions and 441 towns. Current challenges are how to follow-up and evaluate those patients in the clinical settings. The longer the therapy is postponed, the more side effects and the higher probability of drug resistance are going to occur. It remains unclear, therefore, when HAART regimen should be started in the HIV/AIDS population in China.
\end{abstract}

Keywords: HIV/AIDS, needle sharing, unprotected sex, HAART, China CARES program.

\section{INTRODUCTION}

Compared with other areas with high HIV infection rate in the world, the total HIV infection rate in China is relatively low. Nonetheless, in light of China's vast territory and large population, the potential infection risk must be taken into account seriously. The clinical features of the HIV/AIDS epidemic in China include the followings [1]:

1. HIV/AIDS has begun to spread from specific groups into the general populations. Since 2001, some areas in China have been confronted with a particularly high mor-

*Correspondence: Yun Zhen CAO

Tel: +86-10-65105182/65105179-22; Fax: 65105179-12;

E-mail: dryunzhencao@yahoo.com

Abbreviations: HCV (Hepatitis C Virus); TB (Tuberculosis); CPC (Communist Party of Chinese); HAART (Highly Active Anti-Retroviral Therapy); STD (SexuallyTransmitted Disease); ARV (anti-retroviral therapy); CDC (Centers for Disease Control and Prevention); HLA (major histocompatibility complex); CAMS (Confederation of Australian Motor Sport); .ADARC (Aaron Diamond AIDS Research Center); NNRTI (Non-Nucleoside Reverse Transcriptase Inhibitor) bidity and mortality due to AIDS.

2. Many risk factors of HIV transmission remain uncontrolled:

1) In the next few years, needle sharing among injection drug users will remain the most common route of transmission for the HIV/AIDS epidemic in China.

2) Unprotected sex is gradually becoming a major route of transmission for the HIV/AIDS in China.

3) With increases in the number of female HIV patients infected through sex, mother-to-child transmission is bound to increase. Since the first case of mother-to-child transmission (MTCT) to be acknowledged in 1995, MTCT rates have been rising year by year. Currently, mother-to-child transmission mainly occurs in areas with severe HIV epidemic $[2,3]$.

3. High HIV-HCV co-infection rates present a major problem [4].

4. The complexities of HIV-TB co-infection must also be addressed. Confronted with a severe HIV/AIDS epidemic, the Chinese government has been making great efforts to reinforce the prevention of HIV/AIDS in recent 
years. After the establishment of China's Medium-\& Longterm Strategy for HIV/AIDS Prevention and Control (19982010) [5] in 1997 and China's Action Plan for Reducing and Preventing the Spread of HIV/AIDS (2001-2005) in 2001[6], the State Council established the State Council Coordinating Committee for HIV/AIDS/STD Prevention and Control in 2004, so as to fortify the leadership and to mobilize all resources towards prevention. This committee is led by Yi, WU member of CPC Central Committee and Vice Premier of the State Council. After using a prevention-oriented strategy for several years, the Chinese government has shifted towards a strategy that empha- sizes both prevention and treatment, which has been a rather complicated and difficult systematic project. In 2003-2004, the Chinese government directly invested 1.2 billion RMB in the prevention and treatment of HIV/AIDS, and is planning to spend 3.86 billion RMB in 2005-2007 to further promote the implementation of HIV/AIDS prevention and treatment in China.

\section{HIV/AIDS PREVENTION AND CONTROL IN THE PAST 20 YEARS}

In the past 20 years, since HIV was first considered as the cause of AIDS to the present, scientific researches

Tab. 1 Drugs used in the treatment of HIV infection [7]

A

\begin{tabular}{|c|c|c|c|}
\hline Brand name & Generic name & Manufacturer name & Approval date \\
\hline Retrovir & zidovudine, AZT, azidothymidine, ZDV & GSK & 19-Mar-87 \\
\hline Videx & didanosine, ddI, dideoxyinosine & BMS & 9-Oct-91 \\
\hline Zerit & stavudine, $\mathrm{d} 4 \mathrm{~T}$ & BMS & 24-Jun-94 \\
\hline Epivir & lamivudine, 3TC & GSK & 17-Nov-95 \\
\hline Videx EC & enteric coated didanosine & BMS & $31-$ Oct-00 \\
\hline Trizivir & abacavir, zidovudine, and lamivudine & GSK & 14-Nov-00 \\
\hline Viread & tenofovir disoproxil fumarate,TDF & Gilead & 26-Oct-01 \\
\hline Emtriva & emtricitabine,FTC & Gilead Sciences & 2-Jul-03 \\
\hline
\end{tabular}

B

\begin{tabular}{llll}
\hline Viramune & nevirapine, BI-RG-587,NVP & Boehringer Ingelheim & $21-J u n-96$ \\
\hline Rescriptor & delavirdine, DLV & Pfizer & $4-$ Apr-97 \\
Sustiva & efavirenz, EFV & BMS & $17-$ Sep-98 \\
\hline
\end{tabular}

C

\begin{tabular}{llll}
\hline Invirase & saquinavir mesylate, SQV & Hoffmann-La Roche & 6-Dec-95 \\
\hline Crixivan & Indinavir, MK-639, IDV & Merck & $13-$ Mar-96 \\
Norvir & Ritonavir, ABT-538 & Abbott & 1 -Mar-96 \\
Viracept & Nelfinavir mesylate, NFV & Agouron & $14-$ Mar-97 \\
Agenerase & Amprenavir, APV & GSK & $15-$ Apr-99 \\
Kaletra & lopinavir and ritonavir & Abbott & $15-$ Sep-00 \\
Reyataz & Atazanavir sulfate, ATV & BMS & $20-$ Jun-03 \\
Lexiva & Fosamprenavir Calcium, FPV & GSK & $20-$ Oct-03 \\
Aptivus ${ }^{-}$ & Tipranavir(TPV) & Boehringer Ingelheim & $22-J u n-05$ \\
\hline
\end{tabular}

D

\begin{tabular}{lll}
\hline Fuzeon & Enfuvirtide, T-20 & Roche \& Trimeris \\
\hline A $)$ 13-Mar-03
\end{tabular}

(A)Nucleoside reverse transcriptase inhibitors (NRTIs); (B)Nonnucleoside reverse transcriptase inhibitors (NNRTIs); (C)Protease inhibitors (PIs); (D)Fusion inhibitors. 
Tab. 2 HAART clinical trail in China

\begin{tabular}{lllll}
\hline Trail period & Cases & Drug & Supporter \\
\hline 1 & $1999.05-2000.05$ & 23 & Combivir + IDV & GSK \\
1 & $2000.05-2001.05$ & 17 & Combivir +ABC & GSK \\
1 & $2001.05-2002.05$ & 13 & Triziver & GSK \\
1 & $2002.05-2003.05$ & 12 & Triziver & GSK \\
2 & $2000.08-2001.07$ & 20 & EFV+ IDV & Merk \\
3 & $2001.04-2003$ & 30 & DDI+D4T+NVP & BMS, BI \\
4 & $2002.10-2005.10$ & 80 & Triziver & GSK \\
\hline
\end{tabular}

and clinical practices for HIV/AIDS prevention and treatment have achieved great success, yet we are still confronted with a number of challenges. Concerning the mechanism of HIV/AIDS as well as prevention and treatment measurements, many difficult problems remain unsolved, such as ARV. The public no longer considers HIV/AIDS a fatal illness. In the mid 1990s, HAART, in which several drugs are combined, heralded a new era for anti-HIV-I therapy. The AIDS mortality rate subsequently declined. Twenty-five drugs have been authorized by FDA or the HIV/AIDS therapy and can be categorized into three kinds, namely nucleoside reverse transcriptase inhibitors, non-nucleoside reverse transcriptase inhibitors and protease inhibitors[7] (Tab. 1).

Adequate treatment for HIV-infected patients is important for controlling the source of infection and preventing HIV re-transmission. Treatment of AIDS patients is the primary means for improving patients' quality of life, prolonging patients' lives, and reducing mortality. Clinical therapy for HIV/AIDS is one of the essential measurements for the prevention and control of HIV/AIDS proposed in China's Medium- \& Long-term Strategy for HIV/ AIDS Prevention and Control (1998-2010).

\section{CLINICAL TRIALS FOR HIV/AIDS TREAT- MENT IN CHINA}

In 1999, China began to implement HAART according to international standards. Before 2003, there were about $150 \mathrm{HIV} / \mathrm{AIDS}$ patients using free imported anti-virus drugs for treatment in HAART clinical trial groups and about $100 \mathrm{HIV} / \mathrm{AIDS}$ patients using imported drugs on their own. China imported thirteen kinds of ARV drugs. These patients, who spent about 100,000 to 120,000 RMB per capita per year on therapy, were treated under doctors' strict follow-up and guidance. They were therefore able to overcome the initial common side effects of the drugs and take medicine on time, which helped them prolong their lives, enhance their quality of life, control viral replication and rebuild their immunity. These results were encouraging. Up to now, nearly 20 patients have been taking medicine for 6 years. Moreover, the areas of the treatment covered have spread from Beijing beginning in 1999 to Shanghai, Guangdong, Henan, Yunnan, Fujian, and Xinjiang. There were four main clinical trial groups (Tab. 2):

1) The first pilot study was conducted in 1999 [8]: "Triple therapy Combivir (AZT and 3TC) plus Indinavir in Chinese individuals with HIV infection" (Tab. 1). There were 23 chronically HIV-infected patients included in this study. In the first year, patients were given Combivir and Indinavir, followed by Combivir and Abacavir, and in the last two years Trizivir (a combination of Abacavir, 3TC and AZT) was administered. Among the 23 patients, 17 are male and 6 are female; mean age is $40.2(40.17 \pm 8$. 28). Among them, 17 were infected through sexual transmission, including four homosexual, and four heterosexual (by spouse). There were four intravenous-drug users (IVDUs) infected due to sharing used syringes; the other two were infected through blood transfusion. Based on the US CDC diagnosis standards for AIDS (1983), 16 of 23 patients were diagnosed with asymptomatic HIV-1 infection, while the other 7 were diagnosed with AIDS (five of the seven AIDS patients had opportunistic infections before treatment, and the CD4+ cell count was < $200 / \mathrm{mL}$ in two patients). The following parameters were examined every three months: HIV-1 RNA in plasma was quantitatively detected by bDNA (branched-DNA magnification technique; Branched-DNA,Company Bell, Quantiplex ${ }^{\mathrm{TM}}$ System 340) with a sensitivity range of $<$ $50 \sim>500,000$ copies $/ \mathrm{mL}$. The cell counts of CD3, CD4 and $\mathrm{CD} 8$ in peripheral blood and the CD4, CD8 subsets of T-lymphocyte were detected with relative counting, (FACS calibur flow cytometer and fluorescent-labeled monoclonal antibodies, Company Beckman-Dickson). Routine blood tests, liver function, renal function, blood lipid and blood glucose were measured. (ABBOTT CD1600£»Hitachi 7170); The drug resistance trial through genotype assay is using the Trugene HIV-1 Genotyping System and OpenGene DNA Sequencing System (Bayer HealthCare, USA).

Of 23 cases, 12 continued taking medicine after completing four years of HARRT. Before therapy, the average CD4+ T cell count and virus load (HIV-1 RNA) of the 23 cases were $372.1 / \mathrm{ml}$ and $525,765.5 \mathrm{copies} / \mathrm{ml}$ in the blood plasma, respectively. After four years' treatment, the average CD4+ T cell count rose to $615.7 / \mathrm{ml}$ and the average HIV-1 RNA level decreased by $5.7 \log 10$ to 1904.8 cop$\mathrm{ies} / \mathrm{ml}$ in the blood plasma. The HIV-1 RNA level in the blood plasma of $75 \%$ patients remained undetected all the time. During the therapy, 19 patients had gastrointestinal tract complications and the amounts of aminotransferase 
of 3 patients increased by a factor of 5 , yet no clinical hepatitis symptoms appeared. Only two patients had to discontinue medication because of hypersensitivity reactions due to drug resistance and taking Abacavir, respectively. In concert with the results from foreign clinical reports, the HARRT regimen achieved distinctive effects of decreasing virus load, increasing CD4+ T cell count, and reducing opportunistic infections among HIV/ AIDS patients. Due to its convenient administration, Trizivir greatly enhanced patients' adherence and improved their quality of life [9].

2) The second clinical trial was conducted in Bejing, Henan Province, Yunnan Province and Xinjiang Uighur Autonomous Region in China in 2001 [10]: "Combination therapy with once a day Stocrin ${ }^{\mathrm{TM}}$ (Efavirenz) and three times a day Crixivan ${ }^{\mathrm{TM}}$ (Indinavir) in HIV-1 infected patients in China". In this open-label study, a combination of EFV and IDV was administered in 20 chronically HIV-1 infected patients. The average age of the subjects was 39 years, and 60 percent $(12 / 20)$ were men. Among 20 patients, 45 percent $(9 / 20)$ were infected through sexual activity, and 40 percent $(8 / 20)$ through blood transfusion. Changes in HIV-1 viral load and immunological parameters were examined longitudinally over a period of 48 weeks. We found that the drug regimen was generally well tolerated, was efficient at reducing HIV-1 plasma viral load, and at increasing total CD4+ T cell counts. The percentage of CD4+ and CD8+ T cell subsets expressing CD38 and HLA-DR activation markers was positively correlated with plasma viral load, suggesting there is a generalized $\mathrm{T}$ cell activation during HIV-1 infection. However, soon after the initiation of treatment, the levels of CD38 and HLA-DR expression on T cells tended to normalize. Based on these observations, it is anticipated that this combination regimen has potential to translate into long-term clinical benefits [11, 12].

3) In May, 2001, the third clinical therapy group, "Early, aggressive therapy of HIV-1 infected adults in China with DDI, D4T and Neveripine (NVP) assessing the effect on viral load and immune function", started with 30 cases, including 11 from Guangdong, 6 from Yunnan, 8 from Henan, 3 from Beijng, 1 from Shanxi and 1 from Shanghai. For 20 patients with effective therapy, the lab studies showed that after 48 weeks of treatment, CD4+ T cell count increased by $177 / \mathrm{mL}$ on average and the average HIV-1 RNA level reduced from 134816 copies/ml before therapy to 196 copies $/ \mathrm{ml}$ in the blood plasma after therapy. Yet according to the lab monitoring results, 10 patients did not show improvements. Compared with previous methods, this method had the advantage of easier administration, fewer administrations per day, and no dietary limitations, which led to good patient compliance.
However, obvious side effects were observed, including severe gastrointestinal reactions, peripheral neuritis, and abnormal hepatic functions.

4) The trial, "Triple therapy Trizivir (AZT, 3TC, Abacavir) in Chinese with HIV infection" began in Oct 2002. Trizivir $^{\circledR}$ is a three-antiretroviral drug with limited clinical experience in Chinese HIV/AIDS patients. Yunnan Infectious Disease Hospital is carrying out an HIV treatment program in collaboration with CAMS and ADARC to evaluate the safety and efficacy of the regimen in HIV-1 infected Chinese patients. 80 HIV/AIDS patients ( 35 female, 45 male) were enrolled into a single-center, open-label study. The mean age was $36.4 \pm 9.3$ years old. HIV infection was acquired through heterosexual transmission $(n=62)$, injection drug use $(n=11)$, homosexual contact $(n=2)$, blood transfusion $(n=3)$, or unknown routes $(n=2)$. Trizivir ${ }^{\mathrm{TM}}$ is supplied as one tablet twice daily for three years. The study is ongoing. To date, 38 and 23 patients have completed $>24$ and 12-24 months of treatment, respectively. At this time, treatment has been interrupted or changed in 19 cases, 17 (89.5\%) due to adverse events. A rebound in viremia was noted in 4 of the 17 patients. All the samples showed resistance to the RT inhibitors. Trizivir ${ }^{\mathrm{TM}}$ treatment has shown potent ARV activity in Chinese HIV/AIDS patients with varying levels of viral load, even those with viremia level above 500,000 copies $/ \mathrm{ml}$. Prolonged treatment has resulted in greater increases in CD4+ counts and improved the control of opportunistic infections. A marked decrease in hematocrit occurred in approximately $10 \%$ of the patients. Overall, Trizivir ${ }^{\mathrm{TM}}$ yielded reasonably good control of HIV replication [13].

\section{IMPLEMENTATION OF THE CHINA NA- TIONAL FREE ANTIRETROVIRAL THERAPY PROGRAM \\ General information about the national free ARV drugs}

It has been two years since the national free ARV therapy was put into effect in 2003. Generally speaking, this urgent and helpful action was quite meaningful to the promotion and extension of the China's AIDS prevention and control project. After 2003, generic drugs made domestically were put on the market. The China Stop, Prevention, and Treatment of AIDS Action Plan (2001-2005) was unveiled, suggesting establishment of general demonstration districts for AIDS prevention and treatment, building up health education, behavior intervention, medical care and consultant care. As of July 30th, 2005, 127 general demonstration districts for AIDS prevention and treatment had started, encompassing 28 provinces and 83.25 million people. All together 19,456 HIV/AIDS patients received free ARV drugs offered by the national government in 159 
regions and 441 towns. The enrollment standard for therapy in each region was in accordance with the enrollment standard of the national free therapy [14]. Eligibility for antiretroviral therapies is based on a combination of clinical (WHO Stage III, IV) and laboratory criteria (CD4 $<200$ cells $/ \mathrm{mm}^{3}$ ). Clinical criteria are identified through a careful physical examination and medical history. Laboratory criteria rely primarily on a CD4 test, or a total lymphocyte count $\left(<1200\right.$ cells $\left./ \mathrm{mm}^{3}\right)$ when a CD4 is not available. Note that a CD4 test is preferable to a lymphocyte count, and CD4 counts should be used when they are available (Tab. 3 ).

There are four pharmaceutical manufacturers involved in the production of the national free ARV drugs, including five kinds of generic drugs (DDI, D4T, AZT, NVP, and IDV). The Ministry of Health imported large amounts of Combivir, 3TC and EFV from GSK and MERCK. In light of the strict control of long-term application of HAART, the approach and the selection of the formula from the National Free AIDS ARV Drug Therapy Manual were based on the drugs that the government could provide for free.

I. First-line Approach for ARV-Naïve Patients: $\mathrm{D} 4 \mathrm{~T}+\mathrm{DDI}+\mathrm{NVP}$ or AZT+DDI+NVP was selected for patients without obvious hepatic diseases (jaundice, ascites, or the increase of baseline serum aminotransferase) and severe peripheral neuropathies.

II. First-line Approach for ARV-Experienced Patients: Due to its milder toxicity and side effects, 3TC was recommended to replace DDI in the original approach so as to improve or avoid toxic and side effects attributed to DDI or the combination of DDI/D4T. Other drugs were left unchanged.

$80 \%$ of patients were given the combined approach of AZT/D4T+DDI+NVP. Among them, the approach containing AZT was most common, accounting for $77 \%$. As 3TC was distributed for free and new therapy guidelines were implemented, the number of patients taking AZT/ $\mathrm{d} 4 \mathrm{~T}+3 \mathrm{TC}+\mathrm{NVP} / \mathrm{EFV}$ gradually increased, and among them, $\mathrm{d} 4 \mathrm{~T}+3 \mathrm{TC}+\mathrm{NVP}$ was the predominant drug combination used.

Factors affecting the therapeutic effects of ARV drugs
1) Patient compliance

Effective AZT requires almost perfect compliance to its complex therapeutic approach. During the course of taking ATR, patients' high compliance was very essential to reduce virus load in the plasma, to enhance immunity function, to maintain the concentrations of therapeutic drugs and to retard the development of the disease. Many reports indicated that only when the compliance increased to $90 \%-95 \%$ or above, the virus replication in the body could be effectively repressed [15]. Therefore, in order to enhance the compliance and achieve ideal therapeutic effects, the main measures were to reduce the amount and times of administration to as few as possible and to select drug combinations with few side effects and convenient administration. However, it proved very difficult to maintain high levels of compliance during therapy. Patients taking ARVs must tolerate several side effect syndromes with varying degrees, from mild to life-threatening. Moreover, ARV therapy requires patients to take different drugs at different times in a day in some cases. Compliance is affected by many factors, such as the patient's psychosocial and economic situations, opportunistic infections, therapeutic approaches (side effects, drug dosage, drug type, dietary restrictions, etc), the quality of the doctor-patient relationship, and accessibility to the clinics. Since compliance varies from patient to patient with different social, economic and cultural backgrounds, the optimal approach is to take each patient's individual situation into account. Research on compliance has been mainly conducted in western developed countries, but little information about compliance in the developing countries is available. China has no data or information concerning patient compliance. At present, it is necessary in general demonstration districts for AIDS prevention and treatment to start health education and behavioral interventions. This includes promotion of HIV-1 voluntary test and counseling, provision of pre- and post-test counseling. Imparting knowledge about ARV drugs, the best time to start HARRT, direct monitoring of ARV therapy, drugs availability, methods of drug administration, side effects of the drugs, necessary lab tests, etc, would enhance compliance and achieve ideal therapeutic effects.

Tab. 3 Criteria for ART in adults/adolescents [14]

\begin{tabular}{lll}
\hline Clinical & Laboratory & Special considerations \\
\hline WHO Stage IV & CD4 $<200$ & In patients with CD4 between \\
WHO Stage III & TLC $<1200 / \mathrm{mm}^{3 *}$ & $200-350$, rapid fall $>30 \%$ in a \\
Symptomatic disease & & year \\
Extrapulmonary TB & & Patients with active TB and CD4 $>200<350 * *$ \\
& & Pregnancy - see "special case-pregnant women" \\
\hline
\end{tabular}


2) Test for HIV drug resistance

Since several kinds of ARV drugs became available and HAART was adopted, anti-HIV/AIDS therapy has achieved good clinical effects, drastically reducing the morbidity and mortality rate of HIV/AIDS patients. However, HIV-1 drug resistance is not only a major obstacle to sustaining HAART in the long-term, but also the main reason for the failure of anti-HIV/AIDS treatment [16]. We collected the sample from 100 patients who started HAART between 1999 and 2003 [17], including 10 cases with therapy more than 5 years of duration, 62 cases with over 2 years duration, and 53 cases with over 1 year duration. Among them, 19 cases showed rebounding viral load, defined by at least 2 successive viral load detections remaining above 500 copies/ml (bDNA) or unchanged CD4+ cell count, and persistence of clinical symptoms. We conducted the drug resistance test at 47 time points for 19 patients. Trugene HIV-1 Genotyping System and OpenGene DNA Sequencing System (Bayer HealthCare $\_$USA) were employed. Through RT-PCR, sample RNA was amplified into target DNA, which was the protease gene fragment of $297 \mathrm{bp}$ and reverse transcriptase gene fragment of $1680 \mathrm{bp}$ in HIV-1 pol gene. After the Clip reaction as well as the mapping, OpenGene DNA Sequencing System combined and analyzed the sequence automatically. By manual proofreading, clinical test reports and lab research reports were obtained. The results suggested that among 19 patients, 3 of them were not detected with drug resistance in the samples at each time point. Among the 16 patients who developed drug resistance after HAART, 14 cases were those in which plasma samples were taken before the therapy. Only one of them indicated resistance to NNRTIs before therapy. The test results of the 16 patients showed that resistance to different drugs had different degrees at different times after the therapy. And among the 11 cases that had been treated previously with NNRTIs, all of them showed resistance to NNRTIs, and nine of them became resistant within one year of administration (Tab. 4).

Drug resistance emerges mainly through two routes: one is the transmission of drug-resistant strains (initial drug resistance); the other is the transformation from HIV-sensitive strains to drug-resistant strains (induced drug resistance). Our results indicated that during the early stages, the majority of HIV/AIDS patients were not drugresistant before therapy in China. Among the 19 clinical cases that had rebounding viral load, three cases were detected not to be drug-resistant. By detailed interviews with patients, it was verified that their administration compliance was poor. They frequently took medicine discontinuously, suggesting that patient compliance should be well understood before clinicians decide to conduct drug resistance testing for the patients. As for these patients with poor compliance, we should adequately supervise the administration of their medicines and re-test the viral load and CD4+ T cell count monthly so that curative effects can be evaluated.

On Aug 20, 2004, we conducted CD4/CD8 and VL tests for 320 patients who had been receiving therapy for more than 6 months in 5 sites: Shangcai County (Guodun and Wenlou), Xincai County of Zhuma City in Henan Province, Suizhou City in Hubei Province and Lixin City in Anhui Province. The results demonstrated that among those receiving therapy for more than half a year, VLs of 141 samples were higher than 500 copies $/ \mathrm{ml}$, including 126 samples that were high enough to qualify for drug resistance testing. There were four drug combinations among those patients, including 1.D4T+ddI+NVP, 2 . $\mathrm{D} 4 \mathrm{~T}+\mathrm{ddI}+\mathrm{EFV}, 3 . \mathrm{AZT}+\mathrm{ddI}+\mathrm{NVP}$, and $4 . \mathrm{AZT}+\mathrm{ddI}+\mathrm{EFV}$. The results indicated that of the 126 samples with viral load $>500$ copies $/ \mathrm{ml}$ after 6 months therapy in these 5 locations, $67.46 \%(85 / 126)$ showed drug-resistance and

Tab. 4 Test results of 19 cases with clinical therapy failure [17]

\begin{tabular}{|c|c|c|c|c|}
\hline \multirow[t]{2}{*}{$\begin{array}{l}\text { No. } \\
\text { (Month) }\end{array}$} & \multirow[t]{2}{*}{ Failure Time } & \multicolumn{3}{|c|}{$\begin{array}{l}\text { Resistance test after therapy } \\
\text { failure }\end{array}$} \\
\hline & & NRTIs & NNRTIs & PIs \\
\hline DP44 & 11 & + & + & - \\
\hline HZ161 & 7 & + & + & + \\
\hline DP11 & 11 & + & + & - \\
\hline DP31 & 8 & + & - & + \\
\hline GX12 & N/A & - & + & - \\
\hline GDM21 & 6 & + & + & - \\
\hline XYJ & $\mathrm{N} / \mathrm{A}$ & + & + & - \\
\hline HJR & N/A & - & + & - \\
\hline YNC091 & 9 & + & - & - \\
\hline YNC103 & 6 & + & - & - \\
\hline YNC170 & 10 & + & + & - \\
\hline YNC180 & 12 & + & - & - \\
\hline B15 & 9 & - & + & - \\
\hline $\mathrm{B} 25$ & 9 & - & + & - \\
\hline B30 & 11 & - & + & \pm \\
\hline HZ39 & 9 & - & + & - \\
\hline DP26 & 2 & - & - & - \\
\hline YNC070 & 12 & - & - & - \\
\hline GYF & N/A & - & - & - \\
\hline
\end{tabular}

+: Highly resistant; -:_Not resistance; \pm : Possibly resistant;

1) The tested single point was not enough to produce resistance to certain drug

2) This point was found in clinical experiment that it could only lead to resistance of some patients to certain drug. 
the percentage was as high as $80.95 \%$ in Guotun of Shangcai County. Cases without viral load reduction were attributed to the emergence of drug-resistant strains in the patient, rendering therapy ineffective for them. Furthermore, the majority demonstrated resistance or primary resistance to NNRTI. Currently, China is conducting ARV therapy on a large scale and NVP is one of very important components. Because of cross drug resistance, drug resistance mutation sites for NVP could lead to resistance to other NNRTIs [18]. Careful attention should be paid by clinical personnel to avoid the spread of strains resistant to these drugs in China. Ideally, all patients having received therapy should be tested with HIV drug resistance since drug resistance test is very helpful for the establishment of rational therapy measurements. However, current HIV drug resistance testing is still unavailable in almost all areas. Therefore, it is crucial for China's HIV/ AIDS therapy to involve drug resistance testing in the HIV/ AIDS clinical routine tests as soon as possible.

3) Follow-up and evaluation are big problems for ARV therapy

Follow-up and evaluation in the clinical setting are pressing problems for ARV therapy. The objectives of the antiretroviral therapy are as follows: 1 . Virological objective: to reduce viral load to the greatest possible extent and to maintain undetectable levels for as long as possible; 2 . Immunological objective: to reestablish immunity function and/or maintain immunity function; 3. Ultimate objective: to prolong life and improve quality of life for the patient. However, currently in China's rural areas, viral load and drug resistance tests are not available either because of lack of equipment, reagents or technology. Regarding immunity testing, the nation tries its best to support it, but there are still some problems beyond the expected effect. Without follow-up and evaluation, the actual curative effect cannot be estimated. Meanwhile, China's first-line drugs have their limitations so that the probability of inducing drug resistance is very high. Without follow-up and evaluation, we have no basis for maintaining or changing measurements. Moreover, China does not have secondline drugs, leading to problems for patients with long-term therapy.

4) When should HAART regimen be initiated for the HIV/AIDS population in China?

When should HAART regimens be started? Obviously, the concept of postponing therapy is being questioned. Evidence suggests that employing more effective and safer new drugs at an earlier stage of therapy (starting therapy when patient's CD4 T cell count $>350 / \mathrm{mm}^{3}$ ) can improve survival, enhance immunity functions, reduce toxicity and drug resistance, and restore function to $\mathrm{T}$ cell subgroups. Early therapy can also reduce the transmis- sion ability of HIV by alleviating viremia and thus benefit public health [19]. Controversy remains regarding how early therapy should be initiated. Although evidence indicates that the immunity functions of HIV-infected patients without HAART decrease progressively, HAART cannot completely restore immune function in some patients. Therefore, currently it cannot be predetermined to what extent the immunity function will be restored by therapy. It also cannot be determined how delayed therapy will influence the restoration of the immunity function. If the ongoing comparative research on early therapy and delayed therapy fails to yield useful conclusions, clinical decisions must rely on the existing evidence. The existing optimal approach still bears some uncertain factors. Our knowledge of the ideal time for starting therapy is limited [20]. Currently, China (and other developing countries) should offer treatment to patients with CD4 counts between 200 to $350 / \mathrm{mm}^{3}$, who are on the verge of clinical AIDS stage. The longer therapy is postponed, the more the side effects and the higher the possibility of drug resistance. China does not have standardized data for Chinese CD4 and CD8 cell counts. Therefore it remains unclear when HAART regimen should be started in the HIV/ AIDS populations in China.

\section{ACKNOWLEDGEMENTS}

We thank the GSK, Merck, BMS and Boehringer Ingelheim pharmaceutical company for their supplied all ARV drug in our for clinical trial study. We also thank the Office of AIDS for fuddling us to set up the clinical training in China.

\section{REFERENCES}

1 State Council AIDS Working Committee office and UN Theme Group on HIV/AIDS in China. A joint assessment of HIV/AIDS prevention, Treatment and care in China (2004). Beijing, 2004

2 Cao, Y, Li G, Wang SY, et al. Epidemiology, crisis and strategy on HIV mother-to-infant transmission in China. Chi J Infect Dis 2002; 20:185-8

3 Zhuang K, Gui X, Su B, et al. High prevalence of HIV infection among women and their children in Henan Province, China. J AIDS 2003; 33:649-50

4 Ying N, Mei S, Li L, et al. Study on the epidemiology and distribution of Human Immunodeficiency Virus-1 and hepatitis C Virus infection among intravenous drug users and illegal blood donors in China. Chinese Journal of Epidemiology 2003; 24:962-5

5 China's Medium- \& Long-term Strategy for HIV/AIDS Prevention and Control (1998-2010). Mministry of Healthy. China. http://www.chinaids.org.cn/zhq/index.asp

6 China's Action Plan for Reducing and Preventing the Spread of HIV/AIDS (2001 - 2005). Mministry of Healthy. China. http:// www.chinaids.org.cn/zhq/index.asp

7 Drugs Used in the Treatment of HIV infection. http://www.fda. gov/oashi/aids/virals.html

8 Cao Y, Zhang F, Mei S, et al. The first clinical trial in China using 
triple therapy Combivir plus Indinavir in patients with HIV-1 infection. Chinese Medical Journal, Electronic Version 2002; 115 (10). http://www.cmj.org.

9 Wang Q, Wei F, Mei S, et al. Assessing the safety and efficacy of HAART regimens over four years of therapy. Chinese Journal of Infectious Diseases. In press.

$10 \mathrm{Li} \mathrm{L}$, Wei F, Mei S, et al. 48 weeks follow-up the virological and immunological outcomes in combination therapy with Efavirenz and Indinavir in HIV-1 infectious in China. Chinese Journal of STD/AIDS Prevention and Control 2002; 8:326-30.

11 Li L, Wei F, Mei S, et al. Virological and immunological outcomes in HIV-1-infected Chinese patients treated with a combination of Efavirenz and Indinavir for 48 weeks. Chinese Medical Journal 2004; 117:347-52

$12 \mathrm{Li} \mathrm{L}$, Wei F, Mei S, et al. The impact of HAART on immunological parameters in HIV-1-infected Chinese, International AIDS Conference, Bangkok, 2004. MOPE A 3010.

13 Zhou Z, Wang L, Wang Y, et al. The safety and efficacy of Trizivir $^{\circledR}$ in the treatment of HIV infection in Yunnan, China for 24 months. New Orleans, Louisiana-: $45^{\text {th }}$ ICAAC Meeting, 2005

14 China Free ART Manual, Division of Treatment and Care,
NCAIDS, CDC, MOH. China. 2005.

15 Paterson DL, Swindells S. Mohr J, et al. Adherence to protease inhibitor therapy and outcomes in patients with HIV infection. Ann Intern Med 2000; 133:21-30.

16 Wegner SA, Wallace MR, Aronson NE, et al. Long-term efficacy of routine access to antiretroviral-resistance testing in HIV type 1-infected patients: results of the Clinical Efficacy of Resistance Testing trial. Clin Infect Dis 2004; 38:723 -30

17 Wei F, Gao L, Mei S, et al. The report of resistance assay after long term HAART from 19 HIV/AIDS cases. Xiamen, China: National Infectious conference, 2005.

18 Lyons FE, Coughlan S, Byrne CM, et al. Emergence of Antiretroviral Resistance in HIV-Positive Women Receiving Combination Antiretroviral Therapy in Pregnancy. AIDS 2005; 19: 63-67.

19 Holmberg SD, Jr Palella FJ, Lichtenstein KA, et al. The Case for Earlier Treatment of HIV Infection. Clin Infect Dis 2004; 39: $1699-704$.

20 Cohen CJ, Boyle BA. Antiretroviral Therapy: The "When to Start” Debates, Editorial Commentary, Clin Infect Dis 2004; 39: 1705-8. 